die Ausgangslage als Seniorpartner einer Großen Koalition, eine vergleichsweise gute Regierungsbilanz, die Oppositionsrolle im Bund, die „Beißhemmungen“ der Mitkonkurrenten und potentiellen Regierungspartner sowie der enorme Popularitätsvorsprung des amtierenden Ministerpräsidenten.

(3) Die CDU musste das schlechteste Wahlergebnis seit 1990 hinnehmen und blieb weit hinter ihren Möglichkeiten zurück. Für diesen Einbruch gibt es sowohl strukturelle als auch hausgemachte Ursachen: mangelnder Rückenwind aus der Bundespolitik, die strategisch ungünstige Ausgangsposition als Juniorpartner einer Großen Koalition, die fehlende Machtperspektive und der sehr defensiv angelegte Wahlkampf.

(4) Die Linke stagniert unter der 20-Prozent-Marke und wurde anders als in den anderen ostdeutschen Ländern erneut deutlich auf den dritten Platz des Parteiensystems verwiesen. Die innerparteilichen Querelen im Land und im Bund verhinderten vermutlich ein besseres Ergebnis.

(5) Die FDP verlor aufgrund des Bundestrends und der eigenen Zerstrittenheit fast drei Viertel ihrer Wähler und steht nunmehr vor existentiellen Herausforderungen. Demgegenüber konnten Bündnis 90/Die Grünen den Rückenwind vom Bund nutzen und erstmals in den Schweriner Landtag einziehen.

(6) Die rechtsextreme NPD schaffte es erneut in den Landtag und verfestigte sich insbesondere in den östlichen Landesteilen weiter strukturell. Ein schnelles Abschmelzen der NPD-Wählerschaft ist von daher nicht zu erwarten.

(7) Die SPD geht gestärkt, die CDU deutlich geschwächt in die Fortsetzung der Großen Koalition. Es bleibt abzuwarten, ob diese gegenüber 2006 veränderten Ausgangsbedingungen Rückwirkungen auf das Koalitionsklima haben werden.

\title{
Die Berliner Abgeordnetenhauswahl vom 18. September 2011: Grün war nur die Hoffnung, die Realität ist rot-schwarz
}

\author{
Oskar Niedermayer
}

\section{Die Ausgangslage}

Die Abgeordnetenhauswahl 2006 war für die beiden seinerzeitigen Regierungspartner SPD und Linkspartei sehr unterschiedlich ausgegangen: Während sich die Sozialdemokraten auf dem 30-Prozent-Niveau stabilisierten, verlor die Linke etwa die Hälfte ihrer Wähler. ${ }^{1}$ Das Wahlergebnis hätte rechnerisch auch eine rot-grüne Koalition ermöglicht. Sehr zum Ärger der Grünen, die als einzige Partei mit absoluten Stimmengewinnen das Wahlergebnis als klaren Regierungsauftrag ansahen, entschied sich die SPD jedoch für eine Fortsetzung von Rot-Rot.

1 Zur Wahl 2006 vgl. Oskar Niedermayer / Richard Stöss, Die Berliner Abgeordnetenhauswahl vom 17. September 2006: Ein „Weiter so" trotz herber Verluste des Koalitionspartners, in: ZParl, 38. Jg. (2007), H. 1, S. $84-100$. 
Obwohl Klaus Wowereit erst im zweiten Anlauf erneut zum Regierenden Bürgermeister gewählt wurde, Kritiker einen schlechten Start in die zweite Legislaturperiode monierten und der Senat zunächst „wie gelähmt“2 erschien, blieb die SPD im ersten Jahr nach der Wahl in der politischen Stimmung in etwa bei ihren Werten, und die Linke konnte sogar leicht zulegen (vgl. auch Abbildung 1). Dann gingen die SPD-Werte jedoch zurück und verschlechterten sich - nach einer kurzen Erholung - im zweiten Halbjahr 2009 nochmals deutlich. Dies wurde auch in den eigenen Reihen nicht nur auf den bundespolitischen Gegenwind, sondern auch auf Klaus Wowereit zurückgeführt, der sich wie ein „Sonnenkönig“ verhalte, „sichtlich die Lust an der Landespolitik verloren“ habe und „die Geschäfte schleifen “ lasse. ${ }^{3}$ Anfang 2010 nahm Wowereit die Kritik an seiner Amtsführung erstmals ernst und machte einen Versuch der Gegensteuerung, der ihm jedoch zunächst nicht gelang. ${ }^{4}$

Die inhaltliche Bilanz des zweiten rot-roten Senats fiel durchwachsen aus. Im Koalitionsvertrag hatte man sich auf eine moderate Fortsetzung des bisherigen Sparkurses, die Förderung von Modellprojekten zur Gemeinschaftsschule, den Einstieg in einen öffentlich geförderten Beschäftigungssektor und die Ablehnung einer weiteren Privatisierung von Landesunternehmen, die Erarbeitung eines Klimaschutzgesetzes und die Verlängerung des Autobahn-Stadtrings durch den Weiterbau der A 100 geeinigt. Verabschiedet wurde eine Schulreform, die nach der Grundschule nur noch zwei Schulformen vorsieht - die Haupt-, Real- und Gesamtschule vereinende Sekundarschule und das Gymnasium -, und es wurde ein öffentlich geförderter Beschäftigungssektor mit sozialversicherungspflichtigen Arbeitsplätzen statt 1-Euro-Jobs eingerichtet. Die Haushaltskonsolidierung hingegen, die zunächst auf einem guten Weg war, wurde 2009 durch die Finanz- und Wirtschaftskrise wieder gefährdet; in der Verkehrspolitik vertagte der Senat aufgrund interner Konflikte wichtige Entscheidungen bis nach der Wahl' Streit auf Eis gelegt. Zudem kam es ab dem Frühjahr 2010 zu heftigen und andauernden Bürgerprotesten, als bekannt wurde, dass die Flugrouten des neuen Großflughafens BBI anders verlaufen sollen als jahrelang verkündet.

Von den Schwierigkeiten der regierenden SPD konnte die zerstrittene Berliner CDU zunächst nicht profitieren. Erst nach einem heftigen Machtkampf zwischen dem Fraktionsvorsitzenden Friedbert Pflüger und dem Parteivorsitzenden Ingo Schmitt, der im Herbst 2008 mit dem Rücktritt der beiden Kontrahenten endete, fand sie unter dem neuen Fraktions- und Parteivorsitzenden Frank Henkel allmählich wieder zur Geschlossenheit zurück. Henkels Strategie der personellen und inhaltlichen Erneuerung der Partei trug 2010 Früchte: Die CDU konnte die SPD im ersten Halbjahr in der Wählergunst überflügeln, und die Grünen kamen ihr gefährlich nahe. Zwar drückte der bundespolitische Gegenwind die CDU im Herbst 2010 wieder etwas nach unten, dafür zogen nun aber die Grünen an der

2 Joachim Fahrun, Der Senat vergisst das Regieren, in: Berliner Morgenpost vom 6. Februar 2007.

3 Armin Fuhrer, Absturz eines Hoffnungsträgers, in: Focus online vom 2. März 2010, http://www. focus.de/politik/deutschland/wahlen-2011/berlin/tid-17430/klaus-wowereit-absturz-eines-hoffnungsträgers_aid_486018.html (Abruf am 4. März 2010).

4 Im März 2010 erreichte Wowereit den absoluten Tiefpunkt seiner Beurteilung durch die Bevölkerung. Vgl. Infratest dimap, BerlinTREND, März 2010.

5 Man verständigte sich darauf, den Weiterbau der A 100 aufzuschieben, und die Zukunft der maroden, vom Mutterunternehmen Deutsche Bahn ausgebluteten und in den Wintern für Chaos sorgenden S-Bahn blieb unklar. 
SPD vorbei und wurden zur stärksten Partei (vgl. Abbildung 1). Die Ära der SPD als dominierende politische Kraft in Berlin schien daher vorbei zu sein.

\section{Der Wablkampf}

Schon direkt nach der Bundestagswahl Ende September 2009 wurde über eine GrüneSpitzenkandidatur von Renate Künast, der Vorsitzenden der Bundestagsfraktion und früheren Fraktionschefin im Abgeordnetenhaus, spekuliert. ${ }^{6}$ Schon vor der Wahl sollen Landesgrüne mit ihr über dieses Thema diskutiert haben, offiziell bestätigt wurde es jedoch nicht, und Künast selbst schwieg eisern. Auf dem Hintergrund SPD-interner Streitigkeiten und

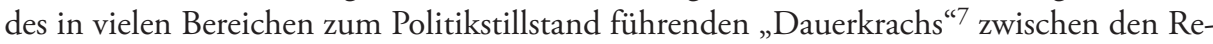
gierungspartnern, zudem gefördert von den guten Umfrageergebnissen und der geschickten Medienstrategie der Grünen, baute sich jedoch spätestens ab Mitte 2010 ein Spannungsbogen auf, der Renate Künast auf Augenhöhe mit Klaus Wowereit katapultierte. ${ }^{8}$ Zum Schluss wurde sie „wie eine Heilsbringerin“ erwartet und bei ihrer spektakulär inszenierten Kür am 5. November 2010 „wie eine Erlöserin"9 gefeiert. Schon kurz nach der Nominierung führten ihre Äußerungen zum flächendeckenden Tempo 30, der Verbeamtung von Lehrern, dem neuen Berliner Flughafen als Regionalflughafen und zur Zukunft der Gymnasien jedoch zu Irritationen und Enttäuschungen. Im weiteren Verlauf des von den Grünen noch nie so extrem personalisiert geführten Wahlkampfes wurde ihre Unterstützung durch die Berliner Wähler immer schwächer, und kurz vor der Wahl wurde sie bei der Bürgermeisterpräferenz sogar vom CDU-Spitzenkandidaten Frank Henkel überholt (vgl. Tabelle 3). Parallel dazu fielen die Grünen auf den letzten Metern in der Wählergunst hinter die CDU auf Platz drei zurück (vgl. Abbildung 1).

$\mathrm{Zu}$ dieser Entwicklung hat sicherlich Renate Künasts Naturell beigetragen, das sie im Wahlkampf mit einer „Kratzbürstigkeit durch die Stadt“ rasen ließ, „die sie immer weiter ... von der Gutmütigkeit eines Wowereit" entfernte. ${ }^{10}$ Eine alleinige Schuldzuweisung an Künast, die als „Spitzenhindernis der Grünen"11 „trotz bester Chancen den Wahlkampf versemmelt "12 habe, ist jedoch nicht gerechtfertigt, denn die Chancen der Partei waren von

6 Vgl. zum Beispiel Joachim Fahrun, Drei gegen Klaus Wowereit, in: Berliner Morgenpost vom 29. September 2009.

7 Gilbert Schomaker / Joachim Fahrun, Die rot-rote Koalition im Dauerkrach, in: Berliner Morgenpost vom 20. Mai 2010.

8 Bei einer Umfrage im September konnte sie Wowereit in der Bürgermeisterpräferenz der Bevölkerung sogar überflügeln. Vgl. Infratest dimap, BerlinTREND, September 2010.

9 Armin Fuhrer, Von nun an ging's bergab, in: Focus vom 18. Juli 2011. Die formelle Wahl Renate Künasts zur Spitzenkandidatin erfolgte erst auf einer Landesmitgliederversammlung am 9. April 2011.

10 Markus Feldenkirchen, Fluch der Vergangenheit, in: Der Spiegel vom 5. September 2011, S. 32 -34 .

11 Uwe Rada, Das Spitzenhindernis der Grünen, in: taz online vom 5. September 2011, http:// www.taz.de/Zwei-Wochen-vor-der-Abgeordnetenhauswahl/!77526/ (Abruf am 5. September 2011).

12 Marcus Jauer, Grüner wird's wohl nicht, in: FAZ.NET vom 13. September 2011, http://www.faz. net/aktuell/politik/wahlkampf-in-berlin-gruener-wird-s-wohl-nicht-11167297.html (Abruf am 14. September 2011). 


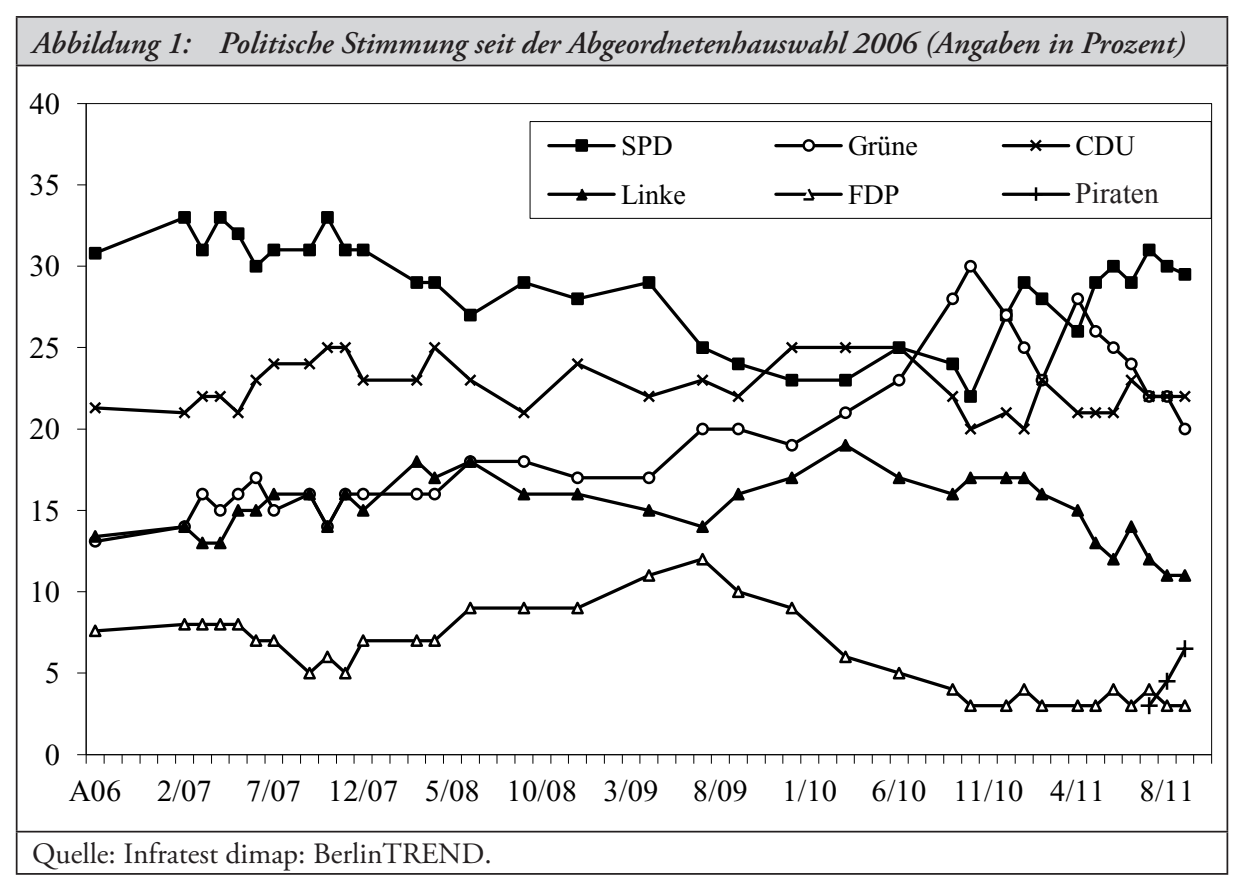

Anfang an weit schlechter als ihre Wahrnehmung: Die Strategie ihres Wahlkampfmanagements wurde durch zwei miteinander verbundene Fehleinschätzungen geprägt. Zum einen unterschätzte man den Einfluss externer Faktoren auf die Berliner Umfrageergebnisse der Grünen: Die beiden kurzen Höhenflüge waren zum großen Teil durch die bundespolitische Diskussion und die nachfolgende Regierungsentscheidung zur Laufzeitverlängerung der Atomkraftwerke sowie die Auseinandersetzungen um „Stuttgart 21“ im Herbst 2010 und die japanische Reaktorkatastrophe in Fukushima im Frühjahr 2011 geprägt und sagten daher wenig über die Chancen der Berliner Grünen aus, die SPD aus eigener Kraft zu schlagen. Zum anderen unterschätzte man die Probleme, die eine in der Euphorie über die guten Umfragewerte gewählte und durch die bundespolitische Diskussion gestützte „Volkspartei“-Strategie einem Teil der Berliner Funktionäre, Mitglieder und Wähler der Grünen bereiten würde. Die jahrelangen Grabenkämpfe zwischen „Fundis“ und „Realos“ um die Ausrichtung der Partei schienen ausgestanden. Unter dem Motto „Wir wollen Volkspartei werden" 13 wurde eine Strategie der Öffnung über das angestammte grüne Milieu hinaus verfolgt. Dafür stand sowohl die Person der Spitzenkandidatin als auch der Slogan der Kampagne „Eine Stadt für alle“. Ausfluss dieser Strategie war zudem die Auffassung, den Wahlkampf inhaltlich möglichst breit anzulegen und unter dem Motto „da müssen wir ran" eine Vielzahl Berliner Probleme und Versäumnisse zu thematisieren. Damit geriet Künast jedoch zum einen immer mehr in die Rolle der die Stadt madig machenden „Meckerliese“, und zum anderen wurde nicht deutlich, für welche zukunftsgerichtete, genuin grüne Vision von Berlin sie eigentlich steht. Koalitionspolitisch implizierte die

13 Vgl. das Interview mit der Landesvorsitzenden Bettina Jarasch, in: Berliner Morgenpost vom 13. März 2011. 
Volkspartei-Strategie das Offenhalten unterschiedlicher Bündnisoptionen. Daher verkündete Künast schon lange vor ihrer offiziellen Nominierung, man werde „in Berlin keinen Wahlkampf machen, der dazu führt, dass die CDU als denkbarer Partner von vornherein ausfällt“, obwohl sich die Grünen ein Zusammengehen mit der SPD „am ehesten vorstellen“ könnten. ${ }^{14}$ Für viele Berliner Mitglieder und Wähler der Grünen gibt es jedoch „eine so fundamentale Distanz zu den Christdemokraten“, dass eine Koalition einem „Kulturbruch" gleichkäme. ${ }^{15}$ Als in der Schlussphase des Wahlkampfs deutlich wurde, dass die Grünen ihr im Wahlprogramm einstimmig beschlossenes Ziel einer Landesregierung unter Führung einer Regierenden Bürgermeisterin Renate Künast wenn überhaupt, dann nur noch in einer Koalition mit der CDU verwirklichen konnten, verweigerten Vertreter des linken Flügels Künast für diesen Fall öffentlich die Unterstützung. Als man dann noch vermutete, dass die Piratenpartei zunehmend linksalternative Stimmen von den Grünen abziehen würde, zog Renate Künast am 8. September im TV-Duell mit Klaus Wowereit die Notbremse und verkündete: „Ich werde meiner Partei nicht vorschlagen, eine Koalition mit der CDU einzugehen. "16 Zudem versuchte der Fraktionsvorsitzende Volker Ratzmann inhaltliche Standfestigkeit zu demonstrieren, indem er den von den Grünen abgelehnten Weiterbau der A 100 als nach der Wahl nicht verhandelbar erklärte.

Der große Vorsprung, den die SPD am Wahlkampfende wieder vor den Grünen hatte, war natürlich nicht nur auf die Wahlkampfprobleme der Grünen zurückzuführen. Deren guten Werte im Herbst 2010 hatten für die „träge Hauptstadt-SPD“ eine „pädagogische Wirkung "17, und für Klaus Wowereit bedeutete die Kandidatur Renate Künasts den entscheidenden Kick, um seinen Kampfeswillen wieder zu wecken. Er begann eine Kieztour, auf der er im direkten Kontakt mit den Wählern seine Menschenfischer-Qualitäten ausspielte, setzte aber nicht nur auf eine reine Charmeoffensive, sondern bezog in seinen Reden und Interviews zu bestimmten Sachfragen auch klar Stellung, zum Beispiel zu den auch in seiner eigenen Partei umstrittenen, von ihm selbst befürworteten großen Verkehrsinfrastrukturprojekten A 100 und dem neuen Großflughafen. Die SPD-Plakatkampagne setzte hin-

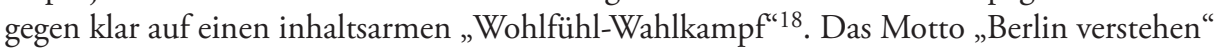
sollte einerseits Emotionen wecken, andererseits aber auch die SPD als einzige Partei stilisieren, die für das gesamte Berlin stehe und in der Lage sei, die Gegensätze der Stadt zusammenzuführen, und die Wowereit-Plakate mit den „menschliche Nähe“-Motiven in Alltagssituationen setzten auf die persönliche Sympathie als derjenigen Imagedimension, auf der Wowereit seinen Kontrahenten am stärksten überlegen war. Koalitionsstrategisch wurde im Wahlkampfverlauf immer deutlicher, dass die SPD-Spitze dem Wunschziel der

14 Jochen Gößmann / Gunnar Schupelius / Felix Frieler, Schwarz-Grün in Berlin ist möglich, in: B.Z. vom 17. Oktober 2010, http://www.bz-berlin.de/aktuell/berlin/schwarz-gruen-in-berlin-istmoeglich-article1008762.html (Abruf am 18. Oktober 2010).

15 Gerd Nowakowski, Erst reden, dann denken, in: Der Tagesspiegel vom 7. Februar 2011.

16 Hannah Beitzer / Thorsten Denkler, Roter Buddha, grüne Friedenstaube, in: sueddeutsche.de vom 9. September 2011, http://www.sueddeutsche.de/politik/tv-duell-in-berlin-roter-buddha-gruenefriedenstaube-1.1140805 (Abruf am 9. September 2011).

17 Hajo Schumacher, In Berlin herrscht keine Wechselstimmung, in: Berliner Morgenpost vom 9. Dezember 2010.

18 Felix Berth, Sachverstand zweitrangig, in: sueddeutsche.de vom 19. September 2011, http://www. sueddeutsche.de/politik/berlin-nach-der-wahl-eine-waehleranalyse-sachverstand-zweitrangig-1.1146140 (Abruf am 19. September 2011). 
Parteimehrheit nach Rot-Grün folgen wollte, auch wenn man die anderen Koalitionsoptionen bis zum Schluss nicht kategorisch ausschloss.

Die Linkspartei hingegen musste sich im Wahlkampf einerseits gegenüber ihrem NochKoalitionspartner profilieren und andererseits mangels Alternative um eine Fortsetzung der rot-roten Koalition kämpfen. Unter dem Motto „Das soziale Berlin“ wollte man deutlich machen, das „Original“ unter den linken Parteien zu sein, und setzte daher programmatisch auf „richtig viel Staat“ ${ }^{19}$. Im Blick war dabei vor allem die Wählerklientel im Ostteil der Stadt. ${ }^{20}$ Weder die propagierten Inhalte noch der sachkompetente, aber wenig mitreißende Spitzenkandidat Harald Wolf konnten jedoch der Partei Aufmerksamkeit verschaffen und die Wähler davon überzeugen, dass die Linke „der Motor für die positiven Entwicklungen der Stadt“21 war. Zudem hatte die Partei mit starkem bundespolitischen Gegenwind zu kämpfen: Der „frivole Umgang“ der Bundesspitze „mit geschichtspolitischen Themen wie Mauerbau, Stalinismus, Diktaturen“ wirkte im Berliner Wahlkampf „unmittelbar parteischädigend“22. Die Linke geriet somit in eine Abwärtsspirale aus abnehmender Wählerunterstützung und zunehmender Frustration, aus der sie sich nicht mehr befreien konnte.

Auch die CDU hatte im Wahlkampf ein strategisches Problem: Einerseits wollte sie deutlich machen, dass sie „die einzige bürgerliche Alternative zu drei linken Parteien in der Stadt "23 sei, andererseits schien bis in die Schlussphase des Wahlkampfs hinein GrünSchwarz als Machtperspektive nicht ausgeschlossen zu sein, so dass man die Grünen nicht zu sehr verprellen durfte. Zudem hatte Frank Henkel drei Jahre lang daran gearbeitet, „die Partei inhaltlich rundzuerneuern und vorsichtig in die politische Mitte zu bugsieren "24. Daher war ein inhaltlich allzu konfrontativ-zugespitzter Angriffswahlkampf keine Option. So musste zum Beispiel auch auf die Serie von nächtlichen Autobrandstiftungen Mitte August eine Antwort gefunden werden, die das zu den Kernkompetenzen der CDU zählende Thema Kriminalität und innere Sicherheit zur Mobilisierung der eigenen Anhänger nutzte, ohne von dem angestrebten neuen, großstädtisch-liberalen Image wieder in das der traditionellen Law-and-Order-Partei zurückzufallen. Angesichts der nach wie vor großen Vorbehalte gegen die Partei in der Bevölkerung ${ }^{25}$ und der geringen Bekanntheit ihres Spit-

19 Werner van Bebber, Linke will wieder richtig viel Staat, in: Der Tagesspiegel online vom 17. Januar 2011, http://www.tagesspiegel.de/berlin/linke-will-wieder-richtig-viel-staat/3705552.html (Abruf am 18. Januar 2011).

20 So versuchte die Linke zum Beispiel die im Wahlkampf durch Mietsteigerungen hochkochende Mietenproblematik für sich zu nutzen, indem sie sich als Schutzmacht gegen „Wild-West bei den Mieten" stilisierte, obwohl man seit zehn Jahren für die Wohnungspolitik im Senat die Mitverantwortung trug.

21 So der Fraktionschef Udo Wolf, in: Lars von Törne, Linke zieht Erfolgsbilanz, Wowereit greift an, in: Der Tagesspiegel vom 25. August 2011.

22 Mechthild Küpper, Gib dem Affen Graubrot, in: faz.net vom 11. September 2011, http://www. faz.net/-024snt (Abruf am 12. September 2011).

23 Interview mit Frank Henkel, in: Berliner Morgenpost vom 6. Januar 2011.

24 Robert Birnbaum / Werner von Bebber, Die Berliner CDU ist wieder wer, in: Der Tagesspiegel online vom 18. September 2011, http://www.tagesspiegel.de/politik/wahlen-2011/die-berlinercdu-ist-wieder-wer/4621898.html (Abruf am 19. September 2011).

25 Kurz vor der Wahl traute nur ein gutes Drittel der Berliner „der CDU in Berlin wieder zu, Verantwortung für die Stadt zu übernehmen “. Infratest dimap, Abgeordnetenhauswahl Berlin 2011. Eine Analyse der Wahl vom 18. September 2011, Berlin 2011, S. 28. 
zenkandidaten (vgl. Tabelle 3) ist der im Wahlkampf erzielte leichte Aufwind (vgl. Abbildung 1) durchaus als Erfolg der Wahlkampfstrategie zu werten.

Die FDP spielte in den Strategieüberlegungen der CDU keine Rolle. ${ }^{26}$ Dies war ihr auch bewusst. Sie sah sich als „Alleinkämpfer“, als einzige noch auf Freiheit und Selbstverantwortung setzende Kraft, und wollte im Wahlkampf als Gegengewicht zu den „vier anderen sozialdemokratisierten Parteien" punkten. ${ }^{27}$ Die Berliner FDP konnte jedoch dem negativen Bundestrend nichts entgegensetzen: Der neue, in Berlin völlig unbekannte Fraktions- und Parteivorsitzende Christoph Meyer wirkte im Wahlkampf „sichtlich überfordert" ${ }^{\text {"28, }}$, und der Landesverband erhielt von den Wählern die mit Abstand schlechteste Bewertung der FDP in einem Bundesland überhaupt, so dass es nicht überraschte, dass zwei Drittel der Bevölkerung der Ansicht waren, die FDP werde in Berlin als Partei nicht mehr gebraucht. ${ }^{29}$ Es half ihr auch nicht, dass sie in den letzten Tagen vor der Wahl mit populistischen Parolen die Wahl in Berlin zur Abstimmung über den Euro-Rettungskurs der Bundesregierung machen wollte.

Neben den bisher angesprochenen fünf Parteien hatten bis zum Ablauf der Meldefrist für die Wahlen zum Abgeordnetenhaus (AHW) und zu den Bezirksverordnetenversammlungen (BVV) am 18. Mai 2011 weitere 33 Parteien beziehungsweise politische Vereinigungen eine Beteiligungsanzeige abgegeben, eine davon wollte nur bei den BVV-Wahlen kandidieren. Für 20 politische Vereinigungen, die weder an der letzten AHW 2006 noch an der letzten Bundestagswahl 2009 in Berlin teilgenommen hatten, war festzustellen, ob sie eine Partei im Sinne des Parteiengesetzes waren. Diese Entscheidungen wurden vom Landeswahlausschuss am 1. Juni getroffen, der für drei Vereinigungen den Parteienstatus nicht feststellte. Somit konnten 34 Parteien für die AHW und eine weitere für die BVVWahlen ihre Wahlvorschläge in Form einer Landesliste beziehungsweise einer oder mehrerer Bezirkslisten bis zum 12. Juli 2011 einreichen. In seiner Sitzung vom 22. Juli 2011 ließ der Landeswahlausschuss letztendlich 22 Parteien zur Abgeordnetenhauswahl zu, davon 15 mit Landeslisten und sieben mit einer oder mehreren Bezirkslisten. ${ }^{30}$

Unter den Kleinparteien stach in der heißen Wahlkampfphase eine hervor: die Piratenpartei. ${ }^{31}$ Mitte August zum ersten Mal in den Umfragen aufgetaucht, wuchs ihre Unterstützung sehr rasch, und kurz vor der Wahl wurde ihnen der Einzug ins Abgeordnetenhaus prognostiziert. Diese beispiellose Karriere verdanken sie einer ganzen Reihe von - meist

26 Vgl. Frank Henkel, „Diese Yuppie-Boygroup braucht in Berlin niemand“, in: Margarete van Ackeren / Olaf Opitz / Frank Thewes, Adieu Paris, bonjour tristesse, in: Focus vom 22. August 2011.

27 Christina Brüning, FDP tritt mit Christoph Meyer an, in: Berliner Morgenpost vom 9. April 2011.

28 Thorsten Jungholt / Christine Kensche, FDP-Wahlkampf am Rande der Bedeutungslosigkeit, in: Die Welt online vom 16. September 2011, http://www.welt.de/politik/deutschland/article13607433/FDP-Wahlkampf-am-Rande-der-Bedeutungslosgkeit.html (Abruf am 17. September 2011).

29 Vgl. Forschungsgruppe Wahlen e.V., Wahl in Berlin. Eine Analyse der Wahl zum Abgeordnetenhaus vom 18. September 2011, Mannheim 2011, S. 26.

30 Vgl. die Pressemitteilungen der Landeswahlleiterin vom 18. Mai, 1. Juni und 22. Juli 2011. Die BVV-Wahlen werden im Folgenden nicht weiter behandelt.

$31 \mathrm{Zu}$ dieser Partei vgl. Oskar Niedermayer, Erfolgsbedingungen neuer Parteien im Parteiensystem am Beispiel der Piratenpartei Deutschland, in: ZParl, 41. Jg. (2010), H. 4, S. 838 - 854. 
berlinspezifischen - Gründen: (1) Sie hatten eine sehr gute Ausgangsposition, denn Berlin ist nicht erst seit 2011 eine Piraten-Hochburg. Die Partei erreichte 2009 bei der Bundestagswahl mit 3,4 Prozent das bei Weitem beste Wahlergebnis aller Bundesländer, und der Berliner Landesverband war mit fast 1.000 Mitgliedern schon vor der heißen Wahlkampfphase der mit Abstand stärkste Landesverband. (2) Als Stadtstaat bietet Berlin die Möglichkeit, mit relativ geringem Ressourcenaufwand einen flächendeckenden offline-Wahlkampf (zum Beispiel durch Plakatierung) zu führen und damit neben der online mobilisierbaren Kernklientel auch Randwähler zu erreichen. (3) Inhaltlich sind die Piraten in Berlin deutlich über ihren Markenkern - die Netzpolitik - hinausgegangen und haben vor allem die Forderung nach mehr Transparenz in der Politik in den Mittelpunkt gestellt, ein Thema, das in Berlin auf offene Ohren traf. ${ }^{32}$ (4) Das Auftauchen einer neuen, unkonventionellen Partei hatte einen hohen Nachrichtenwert, so dass sich eine Aufwärtsspirale aus zunehmender Medienunterstützung und sich verbessernden Umfrageergebnissen entwickelte. (5) Je näher die Piraten der Fünf-Prozent-Hürde kamen, umso attraktiver wurden sie für Wähler, die aus Unzufriedenheit mit den etablierten Parteien bisher entweder gar nicht oder eine der Kleinstparteien gewählt hatten und nun damit rechnen konnten, dass ihre Stimme etwas bewirkt. (6) Als letzter Punkt kam hinzu, dass sich ein Teil der linksorientierten Grünen-Klientel aus Enttäuschung über die Grünen-Spitze, die sich im Wahlkampf zu angepasst, zu sehr als seriöse Partei der Mitte gab und sogar eine Koalition mit der CDU nicht ausschloss, der neuen „Aufmüpfigkeitspartei“33 zuwandte.

\section{Das Wahlergebnis}

Der teuerste Wahlkampf, den Berlin bisher erlebt hatte ${ }^{34}$, endete am Wahlabend mit gefühlten und realen Gewinnern und Verlierern (vgl. Tabelle 1). Die SPD verlor zwar 2,5 Prozentpunkte, wurde jedoch mit 28,3 Prozent eindeutig stärkste Partei und fühlte sich als Wahlgewinner. Die Grünen konnten 4,5 Punkte zulegen, blieben aber mit 17,6 Prozent deutlich hinter den zumindest in der Anfangsphase des Wahlkampfs geschürten Erwartungen und wurden als Wahlverlierer gehandelt. Die CDU konnte nach ihrem niedrigsten Ergebnis von 2006 um zwei Punkte auf 23,3 Prozent zulegen. Die Linkspartei hingegen verlor 1,7 Punkte und kam auf 11,7 Prozent. Eindeutiger Wahlverlierer war jedoch die FDP, die aus dem Abgeordnetenhaus flog, 5,8 Prozentpunkte einbüßte und mit 1,8 Prozent noch hinter der NPD (2,1 Prozent) landete. Eindeutiger Wahlgewinner waren die Piraten, die mit 8,9 Prozent einen Sensationserfolg feierten. Der Stimmenanteil der nicht im Abgeordnetenhaus vertretenen Parteien betrug diesmal zehn Prozent (2006: 14 Prozent).

32 Für das Transparenzthema sensibilisiert wurden die Berliner durch die Diskussion um die Offenlegung der umstrittenen Verträge zur Teilprivatisierung der Berliner Wasserbetriebe, die Mitte Februar 2011 zu einem erstmals in der Geschichte Berlins erfolgreichen Volksentscheid führte.

33 Matthias Kamann, Renate Künast in unmöglicher Mission, in: Die Welt online vom 14. September 2011, http://www.welt.de/politik/wahl/berlin-wahl/article13602561/Renate-Kuenast-in-unmoeglicher-Mission.html (Abruf am 15. September 2011).

34 Die Wahlkampfetats der Parteien betrugen insgesamt rund fünf Millionen Euro (SPD: 1,7; Grüne 1,1; CDU 1,0; Linke 700.000; FDP 35.000; Piraten 20.000 bis 25.000). 


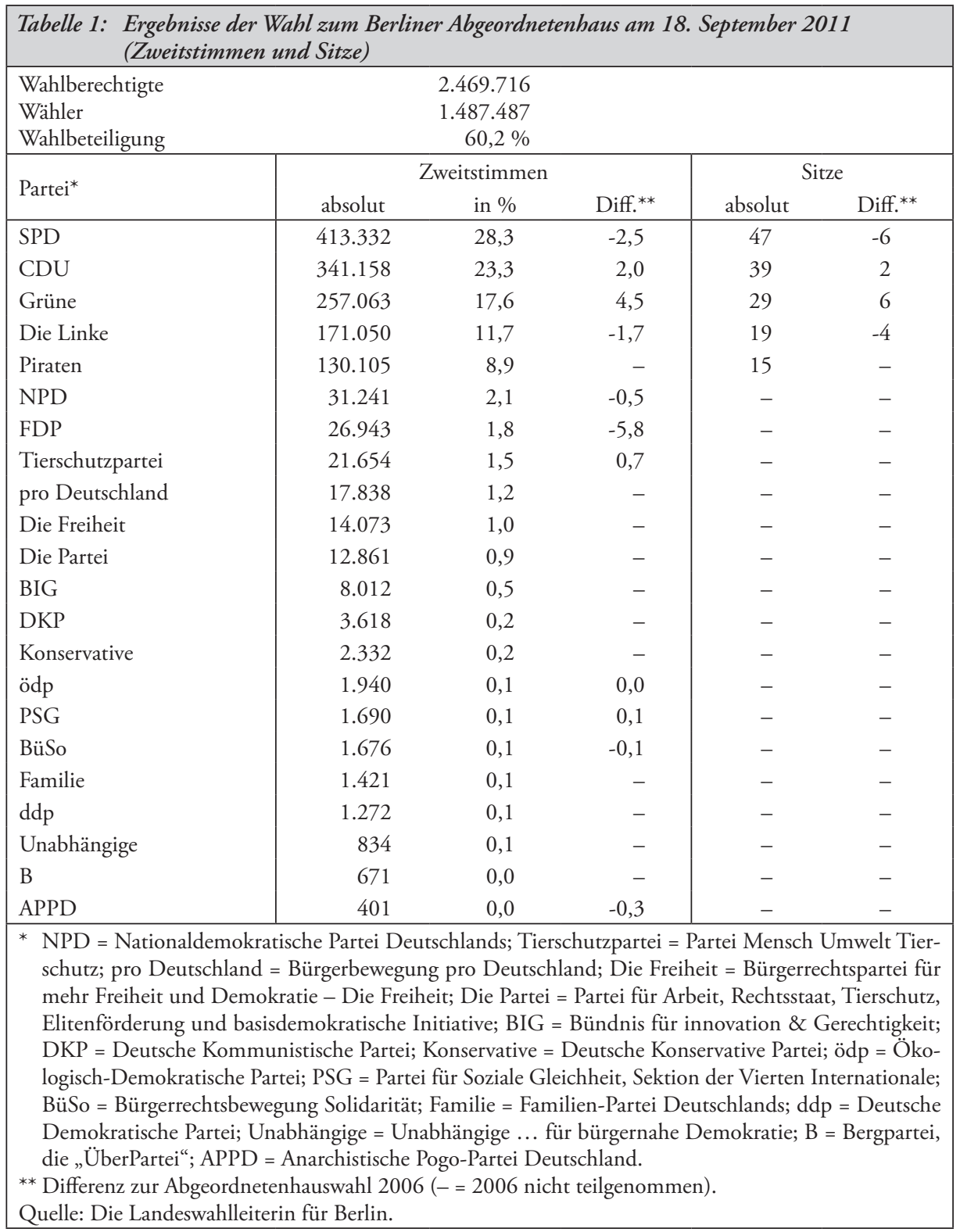

Von den 2.469.716 Wahlberechtigten nahmen 1.487.487 an der Wahl teil. Die Wahlbeteiligung stieg damit von 58,0 Prozent im Jahre 2006 auf 60,2 Prozent. Die Ost-West-Beteiligungsunterschiede blieben weiterhin bestehen, wenn auch in geringerem Maße. ${ }^{35}$ Auch die Verteilung der Parteipräferenzen zeigt für die Mehrheit der Parteien weiterhin deutliche

35 Im Ostteil der Stadt gingen 57,8 Prozent der Wahlberechtigten zur Wahl, im Westteil 62 Prozent. Damit betrug die Differenz 4,2 Prozentpunkte, 2006 waren es 7,3 Prozentpunkte. 
Ost-West-Unterschiede. Am stärksten gilt dies wie in den vorangegangenen Wahlen für die Linkspartei, die im Ostteil wiederum deutliche Einbußen von 28,1 auf 22,7 Prozent hinnehmen musste und im Westteil mit 4,3 Prozent eine eher marginale Partei bleibt. Die CDU ist mit 29,5 Prozent primär im Westen der Stadt verankert, auch wenn sie im Osten etwas stärker (auf 14,2 Prozent) zulegen konnte. Die wenigen noch auf die FDP entfallenden Stimmen zeigen eine ähnliche Differenz (2,3 zu 1,2 Prozent). Auch bei den Grünen ist die Verankerung mit 20,3 Prozent im Westteil der Stadt noch deutlich stärker als im Osten mit 13,5 Prozent. Die einzige Partei, für die dies - seit 2006 - nicht mehr gilt, ist die SPD: Sie erreichte im Westen 27,9 und im Osten 28,8 Prozent. Die Piraten schnitten in OstBerlin etwas besser ab als im Westen (10,1 zu 8,1).

Die Mindestzahl der zu vergebenden Sitze von 130 erhöhte sich bei dieser Wahl - wie 2006 - durch Überhang- und Ausgleichsmandate auf 149. Um die Direktmandate in den 78 Wahlkreisen bewarben sich 626 Kandidaten. Die SPD konnte insgesamt nur noch 47 Sitze erringen (2006: 53), wobei dies vor allem einem deutlichen Rückgang der Direktmandate geschuldet war (2006: 40, jetzt: 33). ${ }^{36}$ Die Linke verlor insgesamt vier Sitze und ein Drittel ihrer Direktmandate. Bei den Grünen ist die von 23 auf 29 gewachsene Mandatszahl ausschließlich darauf zurückzuführen, dass sich die Direktmandate mehr als verdoppelten (5/11) $)^{37}$, und die CDU konnte den Rückgang ihrer Listenmandate (18/14) durch den Zuwachs an Direktmandaten (19/25) überkompensieren, so dass sie insgesamt zwei Sitze hinzugewann. Die 15 Abgeordneten der Piraten sind alle über die Liste ins Parlament eingezogen.

Das Wahlverhalten der verschiedenen sozialen Gruppen hat sich im Vergleich zur Wahl von 2006 bei SPD und CDU wenig geändert: Nach wie vor schneidet die SPD bei den Frauen etwas besser ab; beide Parteien erzielen bei den Wählern ab 60 Jahre die besten Ergebnisse - wobei das CDU-Ergebnis mit zunehmendem Alter kontinuierlich ansteigt und ihr Wählerzuspruch nimmt mit zunehmender Bildung ab (vgl. Tabelle 2). In den Berufsgruppen hat sich die überdurchschnittliche Unterstützung der SPD durch die Arbeiter und der CDU durch die Beamten noch etwas verstärkt, und unverändert wird die CDU von konfessionsgebundenen Wählern deutlich häufiger gewählt als von Konfessionslosen.

Die Linkspartei wurde diesmal etwas stärker von Männern gewählt, und ihr Altersschwerpunkt hat sich nach oben verschoben. Nach wie vor wird die Partei überdurchschnittlich von Arbeitern und Arbeitslosen sowie von Konfessionslosen präferiert. Bei der FDP lässt sich nur noch der Schwerpunkt bei den Beamten und Selbstständigen feststellen. Die Grünen haben ihre Wählerschwerpunkte immer noch bei den jüngeren und mittleren Altersgruppen, den Höhergebildeten und den Beamten sowie Selbstständigen. Ihre Wahlergebnisse sind jedoch über die verschiedenen Alters-, Bildungs- und Berufsgruppen hinweg etwas ausgeglichener als 2006, was darauf hindeutet, dass ihr besseres Wahlergebnis nicht nur durch eine stärkere Mobilisierung der bisherigen Stammklientel, sondern auch durch das Hinzugewinnen neuer Wählerschichten erreicht wurde. Die Piraten wurden überdurchschnittlich von Männern, von Personen jüngeren und mittleren Alters ${ }^{38}$, von Höhergebildeten und von Selbstständigen sowie Arbeitslosen gewählt.

36 Selbst Klaus Wowereit unterlag in seinem Wahlkreis knapp dem CDU-Kontrahenten.

37 Mit Dirk Behrendt, der in seinem Wahlkreis Friedrichshain-Kreuzberg 2 fast die Hälfte der Erststimmen bekam, stellen die Grünen auch den erfolgreichsten Direktmandatsbewerber.

38 Den höchsten Anteil erzielten sie mit 19 Prozent bei den 18- bis 29-jährigen Männern. 


\begin{tabular}{|c|c|c|c|c|c|c|}
\hline \multicolumn{7}{|c|}{ Tabelle 2: Wablentscheidung in sozialen Gruppen (Zeilenprozent) } \\
\hline & SPD & $\mathrm{CDU}$ & Grüne & Linke & Piraten & FDP \\
\hline Gesamt & 28,3 & 23,3 & 17,6 & 11,7 & 8,9 & 1,8 \\
\hline \multicolumn{7}{|l|}{ Geschlecht } \\
\hline männlich & 27 & 24 & 15 & 13 & 11 & 2 \\
\hline weiblich & 30 & 23 & 20 & 11 & 7 & 2 \\
\hline \multicolumn{7}{|l|}{ Alter } \\
\hline 18 bis 29 Jahre & 26 & 15 & 20 & 8 & 15 & 2 \\
\hline 30 bis 44 Jahre & 25 & 19 & 25 & 8 & 12 & 2 \\
\hline 45 bis 59 Jahre & 27 & 22 & 20 & 14 & 9 & 1 \\
\hline 60 Jahre und älter & 34 & 33 & 9 & 15 & 4 & 2 \\
\hline \multicolumn{7}{|l|}{ Bildung } \\
\hline Hauptschule & 34 & 32 & 8 & 11 & 6 & 2 \\
\hline Mittlere Reife & 29 & 27 & 11 & 11 & 8 & 2 \\
\hline Hochschulreife & 27 & 19 & 22 & 10 & 13 & 2 \\
\hline Hochschulabschluss & 24 & 18 & 28 & 14 & 9 & 2 \\
\hline \multicolumn{7}{|l|}{ Berufsgruppe } \\
\hline Arbeiter & 33 & 21 & 10 & 14 & 9 & 1 \\
\hline Angestellte & 30 & 23 & 17 & 13 & 8 & 2 \\
\hline Beamte & 21 & 39 & 21 & 7 & 4 & 3 \\
\hline Selbstständige & 21 & 25 & 24 & 9 & 11 & 3 \\
\hline Arbeitslose & 23 & 12 & 20 & 16 & 16 & 0 \\
\hline $\begin{array}{l}\text { Gewerkschaftlich } \\
\text { organisierte Arbeiter }\end{array}$ & 38 & 15 & 10 & 16 & 8 & 0 \\
\hline \multicolumn{7}{|l|}{ Konfession } \\
\hline katholisch & 25 & 34 & 22 & 3 & 8 & 3 \\
\hline evangelisch & 29 & 33 & 19 & 5 & 6 & 2 \\
\hline keine & 28 & 16 & 17 & 17 & 11 & 2 \\
\hline
\end{tabular}

Betrachtet man die erste Gruppe von kurzfristigen Einflussfaktoren auf die Wahlentscheidung, die Orientierungen gegenüber den Spitzenkandidaten, so zeigt sich der Vorteil, den die SPD durch Klaus Wowereit hatte, schon beim Bekanntheitsgrad: Nach dem Namen des Spitzenkandidaten der jeweiligen Partei gefragt, konnten kurz vor der Wahl sechs Siebtel der Berliner Klaus Wowereit nennen, Renate Künast kannten gut drei Viertel als GrünenKandidatin; und nur die Hälfte der Befragten wusste, dass Frank Henkel Spitzenkandidat der CDU war (vgl. Tabelle 3).

Auch bei den evaluativen Orientierungen der Wähler gegenüber den Kandidaten wurde der Vorsprung Wowereits deutlich: In der allgemeinen Bewertung setzte er sich klar von seinen Herausforderern ab. Dies war vor allem auf seinen großen Sympathievorsprung zurückzuführen, aber auch Führungsstärke und Glaubwürdigkeit wurden ihm in höherem Maße zugeschrieben als seinen Konkurrenten. Lediglich beim wirtschaftspolitischen Sach- 


\begin{tabular}{|l|c|c|c|}
\hline Tabelle 3: Kandidatenorientierungen (Zeilenprozent) \\
\hline \multicolumn{4}{|c|}{ Henkel } \\
\hline (1) Ungestützte Bekanntheit („Wer ist Spitzenkandidat der...?) & 84 & 77 & 50 \\
(2) Allgemeine Bewertung (Mittelwerte, Skala von -5 bis +5) & 1,6 & 0,5 & 0,3 \\
(3) Eigenschaftszuschreibungen* & & & \\
Sympathisch & 77 & 43 & 42 \\
Führungsstark & 74 & 59 & 33 \\
Glaubwürdig & 63 & 58 & 41 \\
Hat großen wirtschaftspolitischen Sachverstand & 33 & 29 & 34 \\
Passt zu Berlin & 49 & 51 & 32 \\
(4) Gewünschter Regierender Bürgermeister** & 65 & 23 & 22 \\
(5) Direktwahl: Wowereit vs. Künast** & 60 & - & 28 \\
Direktwahl Wowereit vs. Henkel & & - \\
\hline * Rating-Skala, das heißt für jede Person wird gesondert gefragt, ob die Eigenschaft zutrifft oder nicht. \\
** Alle drei genannt; fünf Prozent keine der drei Personen, acht Prozent weiß nicht / keine Antwort. \\
*** Nur die beiden genannt, jeweils zehn Prozent keine der beiden Personen. \\
Quelle: (1), (2), (4): Forschungsgruppe Wahlen e.V., a.a.O. (Fn. 29); S. 29, S. 32, S. 30. (3), (5): Infra- \\
test dimap, a.a.O. (Fn. 25), S. 34, S. 32 f. \\
\hline
\end{tabular}

verstand konnte ihn Henkel knapp schlagen. Zusammenfassend waren sechs Siebtel der Befragten der Meinung, dass Wowereit am besten zu Berlin passe, von Renate Künast sagten dies nur die Hälfte und von Frank Henkel nur ein Drittel. Es war daher auch keine Überraschung, dass die Mehrheit Wowereit als Bürgermeister behalten wollte, auch wenn Henkel den Abstand im Vergleich zum CDU-Spitzenkandidaten von 2006, Friedbert Pflüger, deutlich verkürzen konnte.

Insofern war es eine durchaus richtige Entscheidung der SPD, einen personalisierten Sympathiewahlkampf zu führen. Allerdings sollte der unbestreitbare Anteil Wowereits am Wahlerfolg auch nicht überbewertet werden. Nach eigenen Angaben entschlossen sich 32 Prozent der SPD-Wähler, für die Partei wegen ihres Spitzenkandidaten zu stimmen. Dies ist zwar ein deutlich höherer Anteil als bei allen anderen (CDU: 17 Prozent, Grüne: 13 Prozent), jedoch standen bei der Mehrheit der Wähler aller Parteien die Parteikompetenzen in als wichtig angesehenen Sachfragen bei ihrer Entscheidung im Vordergrund ${ }^{39}$, und hier war der Vorsprung der SPD nicht ganz so eindeutig (vgl. Tabelle 4).

Bei dem für die Bevölkerung wichtigsten Thema, der Bildungs- und Schulpolitik, lag die SPD in der Kompetenzzuweisung zwar vorn, jedoch mit geringerem Abstand zu CDU und den Grünen als 2006. Dies galt auch für die Arbeitsmarktpolitik, wo vor allem die Christdemokraten deutlich aufholen konnten. Beim Thema Kriminalitätsbekämpfung, das weit wichtiger war als 2006, konnte die CDU diesmal die SPD überflügeln und in der Wirtschaftspolitik mit ihr gleichziehen. Die Integrationspolitik war dagegen nach Meinung der Bevölkerung bei der SPD am besten aufgehoben und auch die Bekämpfung der Mietsteigerungen, die 2006 noch kein Thema war, wurde der SPD eher zugetraut als den anderen Parteien. Die Kernkompetenz der Grünen, die Umweltpolitik, war für die Wähler nicht 


\begin{tabular}{|l|cccccc|}
\hline \multicolumn{7}{|l|}{ Tabelle 4: Parteikompetenzen bei den wichtigsten politischen Problemen in Berlin } \\
(Angaben in Prozent)*
\end{tabular}

relevant, und die Linkspartei musste in allen wichtigen Politikfeldern außer der Wohnungspolitik Kompetenzverluste in Kauf nehmen. ${ }^{40}$ Ebenso erging es der FDP, die vor allem ihren „Markenkern“, die Wirtschaftskompetenz, verlor.

\section{Regierungsbildung und Oppositionsformierung}

Obwohl das Wahlergebnis sowohl eine rot-grüne als auch eine rot-schwarze Koalition möglich machte - Dreierbündnisse wurden gar nicht erst diskutiert - und die SPD auch beide Parteien zu Sondierungsgesprächen einlud, schien die Regierungsbildung klar vorgezeichnet zu sein: Die Wähler präferierten eindeutig Rot-Grün ${ }^{41}$, der SPD-„,Bauch knurrt(e) nach Rot-Grün“42, und die Medien bilanzierten: „Die Zeichen stehen auf Rot-Grün.“43 Folgerichtig gab es auch keine weiteren Gespräche mit der CDU.

Nach der zweiten Sondierungsrunde zwischen SPD und Grünen am 23. September wurde verkündet, man habe sich bei der wichtigsten Streitfrage, dem Weiterbau der A 100, auf einen Kompromiss geeinigt, der vorsah, dass sich die Koalition für eine Umwidmung

40 Hinzu kam, dass nur noch 17 Prozent (2006: 22 Prozent) der Linken am ehesten zutrauten, für soziale Gerechtigkeit zu sorgen.

41 Danach gefragt, für wen sich die SPD bei einer Wahl zwischen den Grünen und der CDU als Koalitionspartner entscheiden sollte, plädierten kurz vor der Wahl 61 Prozent der Berliner und 76 Prozent der SPD-Anhänger für Rot-Grün, nur 36 beziehungsweise 23 Prozent sprachen sich für die CDU aus. Vgl. Infratest dimap, a.a.O. (Fn. 25), S. 37.

42 Olaf Wedekind, Kopf will Rot-Schwarz, Bauch nicht, in: B.Z. online vom 22. September 2011, http://www.bz-berlin.de/berlin-wahl-2011/kopf-will-rot-schwarz-bauch-nicht-article1278885. html (Abruf am 23.September 2011).

43 Sabine Beikler / Ulrich Zawatka-Gerlach, Die Zeichen stehen auf Rot-Grün, in: Der Tagesspiegel online vom 19. September 2011, http://www.tagesspiegel.de/berlin/die-zeichen-stehen-auf-rotgruen/4624766.html (Abruf 20. September 2011). 
der für die A 100 vorgesehenen Bundesmittel zugunsten der Sanierung und des Ausbaus bestehender Straßen einsetzen würde. Allerdings wurde auch schon deutlich, dass die Grünen bei Klaus Wowereit angesichts der knappen rot-grünen Abgeordnetenhausmehrheit von nur zwei Stimmen noch Überzeugungsarbeit würden leisten müssen, denn „erstens traut der Regierende ihnen das Regieren nicht ohne Weiteres zu, zweitens traut er nicht allen von ihnen" ${ }^{4}$. Nahrung bekam das aufkeimende Misstrauen wenige Tage später, als deutlich wurde, dass die beiden Parteien den Kompromiss in der Frage, was bei einem - wahrscheinlichen - Scheitern der Umwidmungspläne geschehen sollte, konträr interpretierten. Die SPD verkündete: „Lässt sich eine Umwidmung der Bundesmittel nicht erreichen, steht die Koalition zum Weiterbau der BAB 100“, während die Grünen, für die die A 100 zum Prüfstein für ihre Glaubwürdigkeit geworden war, dieser Interpretation vehement widersprachen: „Dem haben wir nicht zugestimmt, und dem werden wir auch niemals zustimmen. " 45 Zwar sprachen sich die Landesvorstände der beiden Parteien am 26. September und die Landesdelegiertenkonferenz der Grünen am 30. September für die Aufnahme von Koalitionsverhandlungen aus, aber die beiden Parteien hatten sich „öffentlich brüskiert. Der Umgang war aggressiv und unfreundlich, das Klima vergiftet “ 46 , und bei der SPD-Führung deutete sich ein Umdenken bezüglich der Koalitionsfrage an. ${ }^{47}$ Am 4. Oktober kam es dann zu einem dritten Sondierungsgespräch, bei dem man, wie es schien, einen neuerlichen Kompromiss fand, über dessen Auslegung die Grünen jedoch bei den am nächsten Tag beginnenden Koalitionsverhandlungen eine erneute Debatte anstießen, was die SPD-Führung dazu veranlasste, die Verhandlungen nach einer knappen Stunde abzubrechen. Selbst Mark Rackles, Sprecher der Parteilinken und „glühender Befürworter von Rot-Grün“, war der Auffassung, dass sich die SPD „auf weitere Interpretationsspielchen ... nicht mehr einlassen “ 48 konnte. Daher beschloss der SPD-Landesvorstand auch noch am selben Abend ohne Gegenstimme und bei nur zwei Enthaltungen, Koalitionsverhandlungen mit der CDU aufzunehmen. Dennoch war es nicht leicht, die SPD-Basis vom linken Flügel von der Sicht des Landesvorstands zu überzeugen, dass die Grünen nicht die notwendige Verlässlichkeit besäßen, um mit ihnen unter der Bedingung der sehr knappen Mehrheit von nur zwei Mandaten eine gesamte Wahlperiode lang regieren zu können. Viele machten sich die Lesart der Grünen zueigen, die Klaus Wowereit vorwarfen, Rot-Grün von Anfang an nicht gewollt zu haben.

44 Stephan-Andreas Casdorff, Alles andere als ideal für Wowereit, in: Der Tagesspiegel online vom 23. September 2011, http://www.tagesspiegel.de/meinung/alles-andere-als-ideal-fuer-wowereit/ 4655070.html (Abruf am 24. September 2011).

45 Vgl. SPD, Gemeinsame Verantwortung für soziale Gerechtigkeit und wirtschaftlichen Erfolg, 26. September 2011, http://www.spd-berlin.de/w/files/spd-diebrie/spd_110926_lv_beschluss_rotgruen.pdf; Bündnis 90/Die Grünen, Wir werden keinem Koalitionsvertrag zustimmen, der den Weiterbau der A100 festschreibt, 28. September 2011, http://www.gruene-berlin.de/thema/stadtentwicklung/wir-werden-keinem-koalitionsvertrag-zustimmen-der-den-weiterbau-der-a100-fest (jeweils Abruf am 7. Februar 2012).

46 Michael Schlieben, Geht's noch, Rot-Grün?, in: Die Zeit online vom 5. Oktober 2011, http:// www.zeit.de/politik/deutschland/2011-10/rot-gruen-berlin (Abruf am 6. Oktober 2011).

47 Vgl. das Interview mit Michael Müller, in: Berliner Kurier vom 1. Oktober 2011.

48 Sabine Beikler / Ulrich Zawatka-Gerlach, Spiel um einen Satz und niemand siegt, in: Der Tagesspiegel online vom 5. Oktober 2011, http://www.tagesspiegel.de/berlin/spiel-um-einen-satz-undniemand-siegt/4689516.html (Abruf am 5. Oktober 2011). 
Auf der Elitenebene gelang der Schwenk zur CDU jedoch reibungsloser, als es nach dem "Trauma der 90er Jahre“ - der von den Sozialdemokraten als „zwölffähriges Martyrium“49 erlebten CDU-SPD-Koalition - zu erwarten gewesen wäre. Dies lag zum einen daran, dass sich die CDU des Jahres 2011 personell und inhaltlich deutlich von der Skandalpartei der Jahrhundertwende unterscheidet; zum anderen stimmte die Chemie zwischen den beiden Verhandlungsführern, so dass relativ schnell gegenseitiges Vertrauen aufgebaut werden konnte. Die am 12. Oktober begonnenen Koalitionsverhandlungen verliefen weitgehend reibungslos und konnten schon am 16. November erfolgreich abgeschlossen werden. Wenn auch die Aussage des SPD-Landes- und Fraktionsvorsitzenden Michael Müller, der 100-seitige Koalitionsvertrag sei „mit roter Tinte geschrieben “50, übertrieben ist, so hat sich die SPD doch in einigen wichtigen strittigen Punkten durchgesetzt: Bei öffentlichen Aufträgen muss jetzt ein auf 8,50 Euro erhöhter Mindestlohn bezahlt werden, für Berlin-Besucher wird eine City-Tax eingeführt, Lehrer werden weiterhin nicht verbeamtet, an der von RotRot eingebrachten Bundesratsinitiative zur Abschaffung des Zwangs für Personen mit doppelter Staatsbürgerschaft, ab dem 18 Lebensjahr für eine der beiden optieren zu müssen, wird festgehalten, Ethik bleibt in den Schulen Pflicht- und Religion freiwilliges Zusatzfach und der von Wowereit favorisierte Neubau der Landesbibliothek kommt. Dagegen konnte die CDU die Abschaffung des Straßenausbaubeitragsgesetzes durchsetzen, die von der SPD gewünschte Bundesratsinitiative zur Einführung eines kommunalen Ausländerwahlrechts abblocken und in der Bildungspolitik die Pflicht zum jahrgangsübergreifenden Lernen abschaffen. Kompromisse wurden gefunden beim Klimaschutz, wo am Ziel der $\mathrm{CO}_{2}$-Reduktion festgehalten wird aber ein konkretes Klimaschutzgesetz nicht auf der Agenda steht, bei der S-Bahn, wo mehrere Zukunftsoptionen geprüft werden, beim Wohnungsneubau, der sowohl von privater Hand als auch durch die landeseigenen Wohnungsbaugesellschaften erfolgen soll, und bei der Rekommunalisierung der teilprivatisierten Wasserbetriebe, die nicht um jeden Preis betrieben werden soll. Einig waren sich die beiden Partner von vornherein zum Beispiel bei den großen Infrastrukturprojekten wie dem Weiterbau der A 100 und dem Großflughafen Berlin-Brandenburg. Zudem wurde eine Erhöhung der Grunderwerbssteuer um 0,5 Prozent beschlossen, um die Einnahmesituation der Stadt zu verbessern, und man verpflichtete sich, die jährliche Ausgabensteigerung auf 0,3 Prozent zu begrenzen, um bis 2016 einen ausgeglichenen Haushalt zu verwirklichen.

Am 21. November 2011 stimmten die Parteitage von SPD und CDU dem Koalitionsvertrag zu - die SPD nach einer zweistündigen Debatte mit 79 Prozent Ja-Stimmen, die CDU einstimmig ohne jegliche Debatte. Am 23. November wurde der Vertrag unterzeichnet und am 24. November wurde Klaus Wowereit im ersten Wahlgang mit 84 von 148 Stimmen zum Regierenden Bürgermeister gewählt. Da die Koalition aus SPD und CDU über 86 Abgeordnete verfügt und zwei Piraten angeblich für Wowereit stimmten, haben $\mathrm{ihm}$ - wenn die anderen Fraktionen geschlossen gegen ihn gestimmt haben - vier Abgeordnete der eigenen Koalition die Gefolgschaft verweigert. Für Wowereit war dies ärgerlich, aber zu verschmerzen, da die Abweichler seine Wahl aufgrund der breiten Mehrheit von Rot-Schwarz nicht gefährdeten.

49 Gerd Nowakowski, Das rot-schwarze Trauma, in: Der Tagesspiegel online vom 5. Oktober 2011, http://www.tagesspiegel.de/berlin/das-rot-schwarze-trauma/4685804.html (Abruf am 6. Oktober 2011).

50 Gut für Berlin und gut für die Menschen, in: Berliner Morgenpost vom 22. November 2011. 


\begin{tabular}{|l|l|}
\hline Tabelle 5: Die Mitglieder des neuen Berliner Senats (Stand: Februar 2012)* \\
\hline Regierender Bürgermeister & Klaus Wowereit (SPD) \\
\hline Senator für Finanzen & Ulrich Nussbaum (SPD) \\
\hline Bürgermeister und Senator für Inneres und Sport & Frank Henkel (CDU) \\
\hline Bürgermeister und Senator für Stadtentwicklung und Umwelt & Michael Müller (CDU) \\
\hline Senatorin für Bildung, Jugend und Wissenschaft & Sandra Scheeres (SPD) \\
\hline Senatorin für Technologie, Forschung und Wirtschaft & Sybille von Obernitz (parteilos) \\
\hline Senatorin für Arbeit, Integration, Frauen & Dilek Kolat (SPD) \\
\hline Senator für Gesundheit und Soziales & Mario Czaja (CDU) \\
\hline Senator für Justiz und Verbraucherschutz & Thomas Heilmann (CDU) \\
\hline * Zur Zusammensetzung der Vorgängerregierung siehe Oskar Niedermayer / Richard Stöss, a.a.O. (Fn. \\
1), S. 98. \\
Quelle: http://www.berlin.de/rbmskzl/rbm/senat/ (Abruf am 7. Februar 2012). \\
\hline
\end{tabular}

Teil der Vereinbarung war eine Ressortaufteilung mit je vier Senatorenposten für die SPD und die CDU, wobei erstere mit dem Regierenden Bürgermeister eine zusätzliche Stimme hat. Mit der Ressortverteilung und der Umbildung von sechs der acht bisherigen Ressorts wurden die Machtverhältnisse sorgfältig austariert und „die Einflusssphären im Sinne künftiger Konfliktvermeidung" ${ }^{\text {"1 }}$ festgelegt.

Mit der Ernennung der Senatoren durch Klaus Wowereit am 30. November und ihrer Vereidigung im Abgeordnetenhaus am 1. Dezember 2011 schien die Regierungsbildung abgeschlossen zu sein. Die Berliner hatten zu dieser Zeit schon weitgehend ihren Frieden mit der vor der Wahl so ungeliebten Koalition gemacht und den beiden Regierungsparteien und ihrem Spitzenpersonal einen Vertrauensvorschuss gewährt. ${ }^{52}$ Bereits am ersten Tag im Amt sah sich der Justiz- und Verbraucherschutzsenator Michael Braun jedoch mit dem Vorwurf konfrontiert, in seiner Eigenschaft als Notar unseriöse Immobilienverkäufe beurkundet zu haben. ${ }^{53} \mathrm{Da}$ in den folgenden Tagen seine Glaubwürdigkeit immer mehr erschüttert wurde und mit dieser neuerlichen Affäre Erinnerungen an die alte, zum Synonym für Filz und Korruption gewordene West-Berliner CDU aufkamen, die das neu gewonnene Wählervertrauen gefährdeten, sah sich die CDU-Führung genötigt, Braun zum Rücktritt zu bewegen. Neuer Justizsenator wurde der stellvertretende CDU-Vorsitzende Thomas Heilmann.

Auch die Opposition blieb von Problemen nicht verschont. Bei den Grünen brach der schon in der Schlussphase des Wahlkampfs wieder deutlich gewordene Konflikt zwischen Realos und Linken, in dem es nicht nur um die inhaltliche Ausrichtung der Partei, sondern auch um persönliche Abneigungen zwischen den Spitzenleuten geht, bei der Wahl der neuen Fraktionsspitze offen aus. Der erstarkte linke Flügel hatte einen der beiden Vorsitzendenposten für sich gefordert, seine Kandidaten waren aber bei der Vorstandswahl am

51 Bernd Matthies, Rot-schwarz geht minimalinvasiv an die Arbeit, in: Der Tagesspiegel online vom 16. November 2011, http://www.tagesspiegel.de/meinung/rot-schwarz-geht-minimalinvasiv-andie-arbeit/5855492.html (Abruf am 17. November 2011).

52 Vgl. Infratest dimap, BerlinTREND, Dezember 2011, Berlin 2011.

53 Vgl. Joachim Fahrun, Ich weiß nichts von Schrottimmobilien, in: Berliner Morgenpost vom 2. Dezember 2011. 
25. Oktober knapp an den bisherigen Vorsitzenden Ramona Pop und Volker Ratzmann gescheitert. Daraufhin schlossen sich die linken Abgeordneten zur „Parlamentarischen Linken“ zusammen, sprachen den gewählten Vorsitzenden die Legitimation ab, die Interessen der Gesamtfraktion zu vertreten, und forderten sie auf, durch Rücktritt für einen linken Kandidaten Platz zu machen. Nachdem dies zunächst zurückgewiesen worden war und auch ein Mediationsverfahren keine Lösung gebracht hatte, machte Ratzmann schließlich den Weg frei. Der linke Flügel schaffte es bei der erneuten Abstimmung am 22. November aber wieder nicht, einen mehrheitsfähigen Personalvorschlag zu präsentieren, so dass die Fraktion zunächst allein von Ramona Pop geführt wird und der Konflikt weiter schwelt.

Angesichts der schweren Krise der Grünen wächst für die Linkspartei die Chance, die angestrebte Oppositionsführerschaft auch tatsächlich zu übernehmen. Auf dem Parteitag am 26. November 2011 wurden die zehnjährige Regierungspraxis und die Vorstandsentscheidungen im Wahlkampf zwar von vielen Rednern „einer posthumen General- und Fundamentalkritik" 54 unterzogen, ein Eklat blieb jedoch aus. Man einigte sich auf eine offensive Oppositionsstrategie, die - auch durch außerparlamentarische Mobilisierungsaktivitäten - wieder stärker die sozialen Interessen der Kernklientel thematisiert.

Während die Berliner FDP zumindest medial im Nirwana verschwand, erfreuten sich die Piraten in den ersten Wochen nach der Wahl eines ungebrochenen und größtenteils unkritisch-positiven Medienhypes: „Die Piraten ... können derzeit beschließen, beraten oder auch nur befinden, was sie wollen. Jede Kleinigkeit wird als Sensation gefeiert, als Sieg von Transparenz, Aufmüpfigkeit, Basisdemokratie und alternativer Authentizität." ${ }^{55}$ Allerdings zeigte sich im Zeitverlauf immer deutlicher eine Reihe von fraktionsinternen Problemen und Konflikten, die bei den Piraten-Abgeordneten die Einsicht wachsen ließ, dass „Piratenkultur und Parlamentsbetrieb nicht gut zueinander passen. " 56 Inwieweit dieses Problem mittelfristig die Wählerunterstützung bröckeln lässt, bleibt abzuwarten.

\section{Zusammenfassung}

(1) Ein Jahr vor der Wahl schien die Ära der SPD als dominierende politische Kraft in Berlin vorbei zu sein. Die CDU hatte sich personell und inhaltlich erneuert, und die Grünen waren in der Wählerunterstützung an ihr vorbeigezogen.

(2) Die mit viel Vorschusslorbeeren bedachte Spitzenkandidatin der Grünen, Renate Künast, konnte sich anfangs auf Augenhöhe mit dem Regierenden Bürgermeister Klaus Wowereit platzieren; ihre Beliebtheit ging jedoch im Wahlkampfverlauf immer mehr zurück, und parallel verloren die Grünen an Wählerunterstützung. Dies lag jedoch nicht allein am Naturell Künasts, die mit Berlin nicht warm wurde, sondern auch an der Un-

54 Werner van Bebber, Berliner Linke wollen wieder linker werden, in: Der Tagesspiegel online vom 27. November 2011, http://www.tagesspiegel.de/berlin/berliner-linke-wollen-wieder-linker-werden/5891802.html (Abruf am 28. November 2011).

55 Jan Thomsen, Diensträder für Piraten, in: Berliner Zeitung online vom 14. Oktober 2011, httb:// www.berliner-zeitung.de/berlin/abgeordnetenhaus-dienstraeder-fuer-piraten,10809148, 11010984.html (Abruf am 15. Oktober 2011).

56 Karin Christmann / Lars von Törne, Piraten nach Mediation etwas klüger, in: Der Tagesspiegel online vom 12. Dezember 2011, http://www.tagesspiegel.de/berlin/piraten-nach-mediation-etwas-klueger/5950710.html (Abruf am 13. Dezember 2011). 
terschätzung des Einflusses externer Faktoren auf die Umfrageergebnisse und an den Problemen, die eine in der anfänglichen Umfrageeuphorie gewählte „Volkspartei“-Strategie und insbesondere das damit einhergehende Offenhalten einer Koalition mit der CDU einem Teil der Grünen und ihren Wählern bereitete.

(3) Die anfängliche Grünen-Euphorie riss die SPD aus ihrer Lethargie und weckte Wowereits Kampfgeist. Mit einem inhaltsarmen, stark personalisierten Wohlfühl-Wahlkampf, der ganz auf den Sympathiebonus des Regierenden Bürgermeisters zugeschnitten war, konnte man wieder Boden gewinnen. Die Linkspartei hingegen vermochte die Wähler weder durch die propagierten sozialpolitischen Inhalte noch durch ihren sachkompetenten, aber wenig mitreißenden Spitzenkandidaten Harald Wolf zu überzeugen und hatte zudem mit starkem bundespolitischen Gegenwind zu kämpfen. Die CDU konnte mit einer auf ein großstädtisch-liberales Image abzielenden Strategie trotz der nach wie vor großen Vorbehalte in der Bevölkerung und der geringen Bekanntheit ihres Spitzenkandidaten im Wahlkampf leicht zulegen. Die FDP jedoch hatte dem negativen Bundestrend weder personell noch inhaltlich etwas entgegenzusetzen, während die neu aufgetauchten Piraten aus einer Reihe von berlinspezifischen Gründen immer stärker wurden.

(4) Die SPD wurde trotz leichter Verluste mit 28,3 Prozent eindeutig stärkste Partei. Die Grünen konnten zulegen, blieben aber mit 17,6 Prozent deutlich hinter den Erwartungen; die CDU verbesserte sich auf 23,3 Prozent, die Linkspartei hingegen ging auf 11,7 Prozent zurück. Eindeutiger Wahlverlierer war die FDP, die mit 1,8 Prozent aus dem Abgeordnetenhaus ausschied; klarer Wahlgewinner waren die Piraten, die mit 8,9 Prozent einen Sensationserfolg feierten.

(5) Das Wahlverhalten der verschiedenen sozialen Gruppen änderte sich nicht wesentlich. Bei den Kandidatenorientierungen zeigte sich überall ein deutlicher Vorsprung Wowereits, während die SPD in der Beurteilung der Sachkompetenzen nicht so eindeutig vorn lag.

(6) Bei der Regierungsbildung schien eine rot-grüne Koalition klar vorgezeichnet zu sein. Die SPD brach jedoch die Koalitionsgespräche sehr schnell ab, weil sie in der Diskussion um die Streitfrage des Weiterbaus der Stadtautobahn A 100 zu der Auffassung gelangte, dass die Grünen nicht die notwendige Verlässlichkeit besäßen, um mit ihnen unter der Bedingung der sehr knappen Mehrheit von nur zwei Mandaten eine gesamte Wahlperiode lang regieren zu können.

(7) Die nachfolgenden Koalitionsverhandlungen mit der CDU verliefen reibungsloser, als es nach den historischen Vorbelastungen zu erwarten gewesen wäre. Nach der Billigung des Koalitionsvertrags durch die beiden Parteitage wurde Klaus Wowereit am 24. November erneut zum Regierenden Bürgermeister gewählt. Dem neuen Senat gehören neben Wowereit vier SPD- und vier CDU-Senatoren an, wobei der neue Justiz- und Verbraucherschutzsenator der CDU wenige Tage nach seinem Amtsantritt aufgrund von Vorwürfen im Zusammenhang mit der Beurkundung unseriöser Immobiliengeschäfte zurücktrat.

(8) Bei den Grünen brach der Konflikt zwischen Realos und Linken in den Fraktionsvorstandswahlen offen aus, und eine Lösung ist noch nicht in Sicht. Die Linke versuchte, die Oppositionsführerschaft zu übernehmen, die FDP war nicht mehr sichtbar, wohingegen die Piraten sich ungebrochener Medienaufmerksamkeit erfreuten. 